\title{
Efficiency of genome-wide association study in random cross populations
}

2 José Marcelo Soriano Viana, ${ }^{* 1}$ Gabriel Borges Mundim, ${ }^{*}$ Hélcio Duarte Pereira, ${ }^{*}$ Andréa Carla

3 Bastos Andrade, ${ }^{*}$ and Fabyano Fonseca e Silva ${ }^{\dagger}$

$4 \quad$ *Federal University of Viçosa, Department of General Biology, 36570-900, Viçosa, MG, Brazil.

$5 \quad{ }^{\dagger}$ Federal University of Viçosa, Department of Animal Science, 36570-900, Viçosa, MG, Brazil.

6 Reference number for data available in public repository:

7 https://dx.doi.org/10.6084/m9.figshare.3201838.version3.

$8 \quad$ REALbreeding private link: https://figshare.com/s/618bee7accd410464232. 
9 Running title: GWAS in random cross populations.

10 KEYWORDS association mapping; GWAS; linkage disequilibrium; inbred lines panel; RIL.

$11{ }^{1}$ Corresponding author: José Marcelo Soriano Viana. Federal University of Viçosa, Department of

12 General Biology, 36570-900, Viçosa, MG, Brazil. E-mail: jmsviana@ufv.br. Telephone:

$13+55(31) 3899-2514$.

14 ABSTRACT Genome-wide association studies (GWAS) with plant species have employed inbred

15 lines panels. Our objectives were to present additional quantitative genetics theory for GWAS, 16 evaluate the relative efficiency of GWAS in non-inbred and inbred populations and in an inbred 17 lines panel, and assess factors affecting GWAS. Fifty samples of 400 individuals from populations 18 with linkage disequilibrium were simulated. Individuals were genotyped for 10,000 single 19 nucleotide polymorphisms (SNPs) and phenotyped for traits controlled by 10 quantitative trait loci 20 (QTLs) and 90 minor genes, assuming different degrees of dominance and heritabilities of 40 and $2180 \%$. The average SNP density was 0.1 centiMorgan and the QTL heritabilities ranged from 3.2 to $2211.8 \%$. To increase the QTL detection power, the additive-dominance model must be fitted for traits 23 controlled by dominance effects but must not be fitted for traits showing no dominance. The power 24 of detection was maximized increasing the sample size to 400 and the false discovery rate (FDR) to $255 \%$. The average power of detection for the low, intermediate, and high heritability QTLs were 9.7, 2632.7 , and $87.7 \%$, respectively. Under sample size of 400 the observed FDR was equal to or lower 27 than the specified level of significance. The association mapping was highly precise. The analysis 28 of the inbred random cross population provided essentially the same results from the non-inbred 29 population. The inbred lines panel provided the best results concerning the low and intermediate 30 heritability QTL detection power, FDR, and mapping precision. The FDR is mainly affected by 31 population structure, compared to relationship information. 


\section{INTRODUCTION}

Association mapping is a high-resolution method for mapping quantitative trait locus (QTL) based on linkage disequilibrium (LD) (Yu and Buckler 2006). Linkage disequilibrium is commonly defined as the non-random association of alleles at two loci carried on the same gamete, caused by their shared history of mutation and recombination (Weir 2008). Association mapping has been successful in detecting genes controlling human diseases and quantitative traits in humans and plant and animal species (Pearson and Manolio 2008; Zhu et al. 2008; Barendse et al. 2007). There are two main association mapping strategies: the candidate gene approach, which focuses on polymorphisms in specific genes that control the traits of interest, and the genome-wide association study (GWAS), which surveys the entire genome for polymorphisms associated with complex traits (Rafalski 2010).

With the advent of high-throughput genotyping and sequencing technologies, breeders have used GWAS to identify genes underlying quantitative trait variation. Compared to QTL mapping, which has precision in the range of $10^{5}$ to $10^{7}$ base pairs ( $\mathrm{Yu}$ and Buckley 2006), the main advantage of GWAS is a more precise identification of candidate genes (Zhu et al. 2008). Another advantage is the use of a breeding population instead of one derived by crossing two inbred or pure lines (Flint-Garcia et al. 2005). However, as highlighted by Weir (2010), the efficiency of GWAS is considerably affected by relatedness and population structure, which can generate spurious association between unlinked marker and QTL. Rafalski (2010) emphasized that the choices of population (due to the degree of LD and genotypic variation), marker density, and sample size are crucial decisions for achieving greater power of QTL detection. Ingvarsson and Street (2011) discussed the influence of population size, extent of LD, trait heritability (precision of phenotyping), and population structure on GWAS efficiency, highlighting that studies with plant species should greatly increase population size to detect QTLs with lower effect (heritability of $1-2 \%)$ 
Yu et al. (2006) proposed a mixed model approach for GWAS analysis called the Q + K (or QK) method, where Q and K are the population structure and kinship matrices, respectively. This method has provided the best results and greatly has improved the control of both type I and type II error rates compared with other methods. Stich and Melchinger (2009) and Yang et al. (2010) compared GWAS methods based on simulated and field data. Based on type I error control and power of QTL detection, they concluded that the mixed model approach using only the kinship matrix (K model) to correct for relatedness was more efficient than the approaches controlling only population structure (Q model) and both population structure and relatedness because the spurious associations could not be completely controlled by population structure. Based on simulated inbred lines panel, Bernardo (2013) demonstrated that his models G and QG were superior to the K and QK, respectively, where G indicates a model that uses genome-wide markers to account for QTLs on background chromosomes. The new approach showed a better balance between power of QTL detection and false discovery rate (FDR). Fraszczak and Szyda (2016) also compared GWAS methods. The genomic selection model was the best method compared to the single SNP, the single SNP with a random polygenic effect (K model), and the CAR score regression.

Recently, many instances of GWAS have been published with plant species, including barley, sorghum, wheat, rice, sugarcane, soybean and particularly maize (Ingvarsson and Street 2011). From the analysis of 271 inbreds genotyped for 28,626 single nucleotide polymorphisms (SNPs), Bernardo and Thompson (2016) calculated chromosomal effects from the effects of SNP alleles carried on the chromosomes. Many chromosome-inbred combinations showed large chromosome $\mathrm{x}$ 77 inbred effects. Pace et al. (2015) carried out a GWAS with 384 maize inbred lines evaluated for 22 78 seedling root architecture traits and genotyped with 681,257 SNPs. They identified 268 marker-trait 79 associations. Some of these SNPs were located within or near (less than one kilo base pairs) to candidate genes involved in root development at the seedling stage. Thirunavukkarasu et al. (2014) 
82

83

high-quality SNPs. The GWAS identified 50 SNPs consistently associated with agronomic traits related to functional traits that could lead to drought tolerance. Thirty-one of the significant SNPs were situated near drought-tolerance genes. Schaefer and Bernardo (2013) used GWAS on a collection of 284 historical maize inbred lines and 39,166 SNPs and identified 19 QTLs for flowering time, 13 for kernel composition, and 22 for disease resistance. However, only two candidate genes were suggested: one regulating days to anthesis and one regulating oil concentration.

Genome-wide association studies with plant species have employed inbred lines panels. Thus, to our knowledge, no information is available on efficiency of GWAS in open-pollinated populations. Because association mapping has been primarily developed for mapping human genes, the available quantitative genetics theory, as that presented by Weir (2008), based on LD and analysis of case-control, is adequate for non-inbred populations. However, there is a lack of quantitative genetics theory for GWAS in inbred populations and inbred lines panels. In this paper we also evidenced the importance of fitting the additive-dominance model for traits showing unidirectional or bidirectional dominance and the additive model when there is no dominance, to achieve high power of QTL detection. We also highlighted the power of QTL detection, the false discovery rate (FDR), and the precision of GWAS. In summary, our objectives were to present additional quantitative genetics theory for GWAS, evaluate the relative efficiency of GWAS in noninbred and inbred populations and in an inbred lines panel, and assess factors affecting GWAS, such as sample size and QTL heritability, effect of substitution, and dominance deviation. Importantly, the results for open-pollinated populations are directly applied to human populations.

\section{MATERIALS AND METHODS}

The following theory aims to prove that a significant association between a SNP and a quantitative trait in an open-pollinated population, in a sample of recombinant inbred lines (RILs), and in an inbred lines panel, after correcting for population structure, depends on the LD between 
the SNP and at least one of the QTLs that affect the trait. Further, we present the parametric values of the average effect of a SNP substitution and LD measure in these association mapping populations, increasing the quantitative genetics knowledge on GWAS previously provided by human geneticists in the context of random population samples, case-control, and family-based 111 studies.

\section{Quantitative genetics theory for GWAS in random cross populations} Consider a biallelic QTL (alleles $\mathbf{B} / \mathbf{b}$ ) and a SNP (alleles $\mathbf{C} / \mathbf{c}$ ) located in the same

114 chromosome, and a population (generation 0) of a random cross species. Assuming LD, the joint

115 gamete and joint genotype probabilities in the population are presented by Weir (2008). The QTL

116 genotypic values are $\mathrm{G}_{\mathbf{B B}}=\mathrm{m}_{\mathrm{b}}+\mathrm{a}_{\mathrm{b}}, \mathrm{G}_{\mathbf{B} \mathbf{b}}=\mathrm{m}_{\mathrm{b}}+\mathrm{d}_{\mathrm{b}}$, and $\mathrm{G}_{\mathbf{b b}}=\mathrm{m}_{\mathrm{b}}-\mathrm{a}_{\mathrm{b}}$, where $\mathrm{m}_{\mathrm{b}}$ is the

117 mean of the genotypic values of the homozygotes, $a_{b}$ is the deviation between the genotypic value

118 of the homozygote of higher expression and $\mathrm{m}_{\mathrm{b}}$, and $\mathrm{d}_{\mathrm{b}}$ is the dominance deviation (the deviation

119 between the genotypic value of the heterozygote and $\mathrm{m}_{\mathrm{b}}$ ). The average genotypic values of

120 individuals with the SNP genotypes $\mathbf{C C}, \mathbf{C c}$, and $\mathbf{c c}$ are

$$
\begin{aligned}
& \mathrm{G}_{\mathbf{C C}}=\frac{1}{\mathrm{p}_{\mathbf{c}}^{2}}\left(\mathrm{f}_{22} \mathrm{G}_{\mathbf{B B C} \mathbf{C}}+\mathrm{f}_{12} \mathrm{G}_{\mathbf{B b C C}}+\mathrm{f}_{02} \mathrm{G}_{\mathbf{b b C C}}\right) \\
& =M+2 q_{c} \kappa_{b c} \alpha_{b}+\left(-2 q_{c}^{2} \kappa_{b c}^{2} d_{b}\right)=M+2 \alpha_{C}+D_{C C}=M+A_{C C}+D_{C C}=m_{c}+a_{c} \\
& \mathrm{G}_{\mathbf{C c}}=\frac{1}{2 \mathrm{p}_{\mathbf{c}} \mathrm{q}_{\mathbf{c}}}\left(\mathrm{f}_{21} \mathrm{G}_{\mathbf{B B C}}+\mathrm{f}_{11} \mathrm{G}_{\mathbf{B b C c}}+\mathrm{f}_{01} \mathrm{G}_{\mathbf{b b C c}}\right) \\
& =M+\left(q_{c}-p_{c}\right) \kappa_{b c} \alpha_{b}+2 p_{c} q_{c} \kappa_{b c}^{2} d_{b}=M+\left(\alpha_{C}+\alpha_{c}\right)+D_{C c}=M+A_{C c}+D_{C c}=m_{c}+d_{c} \\
& \mathrm{G}_{\mathbf{c c}}=\frac{1}{\mathrm{q}_{\mathrm{c}}^{2}}\left(\mathrm{f}_{20} \mathrm{G}_{\text {BB.cc }}+\mathrm{f}_{10} \mathrm{G}_{\text {Bbcc }}+\mathrm{f}_{00} \mathrm{G}_{\text {bbcc }}\right) \\
& =M+\left(-2 p_{c} \kappa_{b c} \alpha_{b}\right)+\left(-2 p_{c}^{2} \kappa_{b c}^{2} d_{b}\right)=M+2 \alpha_{c}+D_{c c}=M+A_{c c}+D_{c c}=m_{c}-a_{c}
\end{aligned}
$$


124 where $p$ is the frequency of the major allele ( $\mathbf{B}$ or $\mathbf{C}), q=1-p$ is the frequency of the minor allele

125 (b or $\mathbf{c}$ ), $\mathrm{f}_{\mathrm{ij}}$ is the probability of the individual with $\mathrm{i}$ and $\mathrm{j}$ copies of the allele $\mathbf{B}$ of the QTL and the

126 allele $\mathbf{C}$ of the SNP (i, j = 2, 1, or 0) (for simplicity, we omitted the superscript ( 0 ) - for generation 0

127 - in all parameters that depend on the LD measure of generation -1),

$128 \mathrm{M}=\mathrm{m}_{\mathrm{b}}+\left(\mathrm{p}_{\mathrm{b}}-\mathrm{q}_{\mathrm{b}}\right) \mathrm{a}_{\mathrm{b}}+2 \mathrm{p}_{\mathrm{b}} \mathrm{q}_{\mathrm{b}} \mathrm{d}_{\mathrm{b}} \quad$ is $\quad$ the $\quad$ population $\quad$ mean, $\quad \kappa_{\mathrm{bc}}=\left[\frac{\Delta_{\mathrm{bc}}^{(-1)}}{\mathrm{p}_{\mathrm{c}} \mathrm{q}_{\mathrm{c}}}\right]$,

$129 \alpha_{b}=a_{b}+\left(q_{b}-p_{b}\right) d_{b}$ is the average effect of a gene substitution, $\alpha_{C}=q_{c} \kappa_{b c} \alpha_{b}$ and

$130 \alpha_{c}=-p_{c} \kappa_{b c} \alpha_{b}$ are the average effects of the SNP alleles, and A and D are the SNP additive and

131 dominance values. $\Delta_{\mathrm{bc}}^{(-1)}=\mathrm{P}_{\mathbf{B C}}^{(-1)} \mathrm{P}_{\mathbf{b c}}^{(-1)}-\mathrm{P}_{\mathbf{B c}}^{(-1)} \mathrm{P}_{\mathbf{b C}}^{(-1)}$ is the measure of LD in the gametic pool of

132 generation -1 (Kempthorne 1957), where $\mathrm{P}^{(-1)}$ indicates a joint gamete probability. Another

133 common measure of LD is the square of the correlation between the values of the alleles at the two

134 loci $\left(\mathrm{r}_{\mathrm{bc}}^{(-1)}\right)$ in the gametic pool of generation -1 (Hill and Robertson 1968). Note that

$135 \Delta_{\mathrm{bc}}^{(-1)}=\mathrm{r}_{\mathrm{bc}}^{(-1)} \sqrt{\mathrm{p}_{\mathrm{b}} \mathrm{q}_{\mathrm{b}} \mathrm{p}_{\mathrm{c}} \mathrm{q}_{\mathrm{c}}}$. The average effect of substituting the allele $\mathbf{C}$ for $\mathbf{c}$ is

$136 \alpha_{\mathrm{SNP}}=\alpha_{\mathbf{C}}-\alpha_{\mathbf{c}}=\kappa_{\mathrm{bc}} \alpha_{\mathrm{b}}$. The dominance deviation for the SNP is $\mathrm{d}_{\mathrm{SNP}}=\kappa_{\mathrm{bc}}^{2} \mathrm{~d}_{\mathrm{b}}$. The other

137 SNP parameters are $\mathrm{m}_{\mathrm{c}}=\mathrm{M}+\left(\mathrm{q}_{\mathrm{c}}-\mathrm{p}_{\mathrm{c}}\right) \alpha_{\mathrm{SNP}}-\left(1-2 \mathrm{p}_{\mathrm{c}} \mathrm{q}_{\mathrm{c}}\right) \mathrm{d}_{\mathrm{SNP}}, \mathrm{a}_{\mathrm{c}}=\alpha_{\mathrm{SNP}}-\left(\mathrm{q}_{\mathrm{c}}-\mathrm{p}_{\mathrm{c}}\right) \mathrm{d}_{\mathrm{SNP}}$,

138 and $\mathrm{d}_{\mathrm{c}}=\mathrm{d}_{\mathrm{SNP}}$. The GWAS and genomic selection models commonly fit the SNP parameters a

139 (values 1, 0, and -1 for SNP genotypes $\mathbf{C C}, \mathbf{C c}$, and $\mathbf{c c}$, respectively) and $\mathrm{d}$ (values 0,1 , and 0 for

140 SNP genotypes CC, Cc, and cc, respectively).

141 Assuming no QTL in LD with the SNP $\left(\Delta_{\mathrm{bc}}^{(-1)}=0\right), \mathrm{G}_{\mathbf{C C}}=\mathrm{G}_{\mathbf{C c}}=\mathrm{G}_{\mathbf{c c}}=\mathrm{M}$. Thus, the

142 identification of the QTL can be based on testing the hypothesis that there is no difference between

143 these genotypic means. Assuming thousands of SNPs, it is necessary to employ a Bonferroni-type 
144 procedure to control the type I error when there are multiple-comparisons, as that proposed by

145 Benjamini and Hochberg (1995). Note that $\alpha_{S N P}=a_{c}+\left(q_{c}-p_{c}\right) d_{S N P}$, where $a_{c}=G_{C C}-m_{c}$,

$146 \mathrm{~d}_{\mathrm{SNP}}=\mathrm{G}_{\mathbf{C c}}-\mathrm{m}_{\mathrm{c}}$, and $\mathrm{m}_{\mathrm{c}}=\left(\mathrm{G}_{\mathbf{C C}}+\mathrm{G}_{\mathbf{c c}}\right) / 2$.

\section{Quantitative genetics theory for GWAS with inbred lines panel}

148 In general, the inbred lines in a panel represent the genetic variability for the traits being

149 assessed. Therefore, an inbred lines panel includes inbreds from distinct populations or heterotic

150 groups. Consider again a QTL (alleles B/b) and a SNP (alleles $\mathbf{C} / \mathbf{c}$ ) located in the same

151 chromosome, and that they are in LD in a population (generation 0). Assuming $\mathrm{n}(\mathrm{n} \rightarrow \infty)$

152 generations of selfing, the (limits of the) probabilities of the inbreds are (for simplicity, we omitted

153 again the superscript (0) - for generation 0 - in all parameters that depend on the LD measure of

154 generation -1$)$

$\mathrm{f}_{22}^{(\mathrm{n})}=\mathrm{f}_{22}+\frac{1}{2}\left(\mathrm{f}_{21}+\mathrm{f}_{12}\right)+\frac{1}{4} \mathrm{f}_{11}+\frac{1}{2}\left(\frac{1-2 \theta_{\mathrm{bc}}}{1+2 \theta_{\mathrm{bc}}}\right) \Delta_{\mathrm{bc}}^{(-1)}$

156

$\mathrm{f}_{20}^{(\mathrm{n})}=\mathrm{f}_{20}+\frac{1}{2}\left(\mathrm{f}_{21}+\mathrm{f}_{10}\right)+\frac{1}{4} \mathrm{f}_{11}-\frac{1}{2}\left(\frac{1-2 \theta_{\mathrm{bc}}}{1+2 \theta_{\mathrm{bc}}}\right) \Delta_{\mathrm{bc}}^{(-1)}$

$\mathrm{f}_{02}^{(\mathrm{n})}=\mathrm{f}_{02}+\frac{1}{2}\left(\mathrm{f}_{01}+\mathrm{f}_{12}\right)+\frac{1}{4} \mathrm{f}_{11}-\frac{1}{2}\left(\frac{1-2 \theta_{\mathrm{bc}}}{1+2 \theta_{\mathrm{bc}}}\right) \Delta_{\mathrm{bc}}^{(-1)}$

$\mathrm{f}_{00}^{(\mathrm{n})}=\mathrm{f}_{00}+\frac{1}{2}\left(\mathrm{f}_{01}+\mathrm{f}_{10}\right)+\frac{1}{4} \mathrm{f}_{11}+\frac{1}{2}\left(\frac{1-2 \theta_{\mathrm{bc}}}{1+2 \theta_{\mathrm{bc}}}\right) \Delta_{\mathrm{bc}}^{(-1)}$

159 where $\theta_{b c}$ is the frequency of recombinant gametes. The haplotypes are $P_{\mathbf{B C}}^{(n)}=p_{b} p_{c}+\Delta_{b c}^{(n)}$,

$160 \quad \mathrm{P}_{\mathbf{B c}}^{(\mathrm{n})}=\mathrm{p}_{\mathrm{b}} \mathrm{q}_{\mathrm{c}}-\Delta_{\mathrm{bc}}^{(\mathrm{n})}, \quad \mathrm{P}_{\mathbf{b C}}^{(\mathrm{n})}=\mathrm{q}_{\mathrm{b}} \mathrm{p}_{\mathrm{c}}-\Delta_{\mathrm{bc}}^{(\mathrm{n})}, \quad$ and $\quad \mathrm{P}_{\mathbf{b c}}^{(\mathrm{n})}=\mathrm{q}_{\mathrm{b}} \mathrm{q}_{\mathrm{c}}+\Delta_{\mathrm{bc}}^{(\mathrm{n})}, \quad$ where

$161 \Delta_{\mathrm{bc}}^{(\mathrm{n})}=\left(\frac{1}{1+2 \theta_{\mathrm{bc}}}\right) \Delta_{\mathrm{bc}}^{(-1)}$. Thus, if there is crossing over $\left(0<\theta_{\mathrm{bc}} \leq 0.5\right)$, the LD in this inbred

162 population is lower than the LD in generation -1. If the SNP and QTL are completely linked ( $\theta_{\text {bc }}$ 
$163=0$ ), the $\mathrm{LD}$ in the inbred population is the same LD in generation -1 . The maximum decrease is

$16450 \%$, achieved with $\theta_{\mathrm{bc}}=0.5$. Compared with the LD in generation 0 , the LD in generation $\mathrm{n}$ is

165

$\Delta_{\mathrm{bc}}^{(\mathrm{n})}=\left[\frac{1}{\left(1+2 \theta_{\mathrm{bc}}\right)\left(1-\theta_{\mathrm{bc}}\right)}\right] \Delta_{\mathrm{bc}}^{(0)}$. Thus, the maximum decrease is approximately $10 \%$, achieved

166 with $\theta_{\mathrm{bc}}=0.25$. In contrast, after $\mathrm{n}$ generations of random crosses

$167 \Delta_{\mathrm{bc}}^{(\mathrm{n})}=\left(1-\theta_{\mathrm{bc}}\right)^{\mathrm{n}+1} \Delta_{\mathrm{bc}}^{(-1)}=\left(1-\theta_{\mathrm{bc}}\right)^{\mathrm{n}} \Delta_{\mathrm{bc}}^{(0)}$. Thus, if $0<\theta_{\mathrm{bc}} \leq 0.5$, the maximum decrease is $100 \%$

168 since $\lim _{\mathrm{n} \rightarrow \infty} \Delta_{\mathrm{bc}}^{(\mathrm{n})}=0$

169 If the panel includes double haploid $(\mathrm{DH})$ lines, the LD for the $\mathrm{DH}$ lines sampled from a

170 population is $\Delta_{\mathrm{bc}}=\Delta_{\mathrm{bc}}^{(0)}=\left(1-\theta_{\mathrm{bc}}\right) \Delta_{\mathrm{bc}}^{(-1)}$. Thus, the LD in a sample of DH lines is greater than the

171 LD in a sample of inbred lines (up to $12.5 \%$ greater when $\theta_{b c}=0.25$ ).

172 For the inbreds sampled from a population, we have

$\mathrm{G}_{\mathbf{C C}}^{(\mathrm{n})}=\frac{1}{\mathrm{f}_{.2}^{(\mathrm{n})}}\left[\mathrm{f}_{22}^{(\mathrm{n})}\left(\mathrm{m}_{\mathrm{b}}+\mathrm{a}_{\mathrm{b}}\right)+\mathrm{f}_{02}^{(\mathrm{n})}\left(\mathrm{m}_{\mathrm{b}}-\mathrm{a}_{\mathrm{b}}\right)\right]=\mathrm{M}_{\mathrm{IL}}+2 \mathrm{q}_{\mathrm{C}} \alpha_{\mathrm{SNP}}^{(\mathrm{n})}=\mathrm{M}_{\mathrm{IL}}+\mathrm{A}_{\mathbf{C} \mathbf{C}}^{(\mathrm{n})}$

$\mathrm{G}_{\mathbf{c c}}^{(\mathrm{n})}=\frac{1}{\mathrm{f}_{.0}^{(\mathrm{n})}}\left[\mathrm{f}_{20}^{(\mathrm{n})}\left(\mathrm{m}_{\mathrm{b}}+\mathrm{a}_{\mathrm{b}}\right)+\mathrm{f}_{00}^{(\mathrm{n})}\left(\mathrm{m}_{\mathrm{b}}-\mathrm{a}_{\mathrm{b}}\right)\right]=\mathrm{M}_{\mathrm{IL}}-2 \mathrm{p}_{\mathrm{c}} \alpha_{\mathrm{SNP}}^{(\mathrm{n})}=\mathrm{M}_{\mathrm{IL}}+\mathrm{A}_{\mathbf{c c}}^{(\mathrm{n})}$

175 where $\mathrm{M}_{\mathrm{IL}}=\mathrm{m}_{\mathrm{b}}+\left(\mathrm{p}_{\mathrm{b}}-\mathrm{q}_{\mathrm{b}}\right) \mathrm{a}_{\mathrm{b}}$ is the inbred population mean, $\alpha_{\mathrm{SNP}}^{(\mathrm{n})}=\left(\frac{1}{1+2 \theta_{\mathrm{bc}}}\right) \alpha_{\mathrm{SNP}}$ is the

176 SNP average effect of allele substitution in the inbred population, and A is the SNP additive value

177 for an inbred line. Assuming no QTL in LD with the SNP, $G_{\mathbf{C C}}^{(n)}=G_{\mathbf{c c}}^{(n)}=M_{I L}$. Notice that

$178 \alpha_{\mathrm{SNP}}^{(\mathrm{n})}=\left(\mathrm{G}_{\mathbf{C C}}^{(\mathrm{n})}-\mathrm{G}_{\mathbf{c c}}^{(\mathrm{n})}\right) / 2$. In the case of DH lines, $\alpha_{\mathrm{SNP}}^{*}=\left(1-\theta_{\mathrm{bc}}\right) \alpha_{\mathrm{SNP}}$. Thus, assuming

$179 \theta_{\mathrm{bc}}=0$, the SNP average effect of substitution is approximately the same in a non-inbred and in an 


$$
\mathrm{P}_{\mathbf{B C}}^{(\mathrm{n})^{\prime}}=\overline{\mathrm{p}}_{\mathrm{b}} \overline{\mathrm{p}}_{\mathrm{c}}+\Delta_{\mathrm{bc}}^{(\mathrm{n})^{\prime}}, \quad \mathrm{P}_{\mathbf{B c}}^{(\mathrm{n})^{\prime}}=\overline{\mathrm{p}}_{\mathrm{b}} \overline{\mathrm{q}}_{\mathrm{c}}-\Delta_{\mathrm{bc}}^{(\mathrm{n})^{\prime}}, \quad \mathrm{P}_{\mathbf{b C}}^{(\mathrm{n})^{\prime}}=\overline{\mathrm{q}}_{\mathrm{b}} \overline{\mathrm{p}}_{\mathrm{c}}-\Delta_{\mathrm{bc}}^{(\mathrm{n})^{\prime}}, \quad \text { and } \quad \mathrm{P}_{\mathbf{b c}}^{(\mathrm{n})^{\prime}}=\overline{\mathrm{q}}_{\mathrm{b}} \overline{\mathrm{q}}_{\mathrm{c}}+\Delta_{\mathrm{bc}}^{(\mathrm{n})^{\prime}}
$$

183 where $\Delta_{b c}^{(n)^{\prime}}=\sum_{i=1}^{N} u_{i}\left[\Delta_{b c_{i}}^{(n)}+p_{b_{i}} p_{c_{i}}\right]-\left(\sum_{i=1}^{N} u_{i} p_{b_{i}}\right)\left(\sum_{i=1}^{N} u_{i} p_{c_{i}}\right)=\bar{\Delta}_{b c}^{(n)}+\overline{p_{b} p_{c}}-\bar{p}_{b} \bar{p}_{c}$ and $u_{i}$ is the

probability of an inbred line belonging to population i. Our simulated data showed that the LD

value in an inbred lines panel tends to be lower than the $\mathrm{LD}$ in each group of inbreds (that are lower than the LD in the base populations) because it is an admixture of positive and negative LD values.

\section{Simulation}

We simulated 50 samples of populations with LD using the software REALbreeding (Viana et al. 2017, 2016, 2013; Azevedo et al. 2015). This software has been developed by the first author using the program REALbasic 2009. Population 1, generation 0, is a composite of two populations in linkage equilibrium. Population 1, generations 10s and 10r10s, were obtained from Population 1, generation 0 , assuming 10 generations of selfing and 10 generations of random crosses followed by 10 generations of selfing, respectively, assuming sample sizes of 100 and 400, respectively. Populations 2, 3, and 4, generation 10s, are also inbred populations (10 generations of selfing) derived from composites of two populations, also assuming a sample size of 100. The parents of populations 2 and 3 were assumed to be non-improved and improved populations, respectively. An improved population was defined as having frequencies of favorable genes greater than 0.5 , while a non-improved population was defined as having frequencies less than 0.5. A composite is a HardyWeinberg equilibrium population with LD for only linked markers and genes. In the case of a composite of two populations in linkage equilibrium, $\Delta_{b c}^{(-1)}=\left(\frac{1-2 \theta_{b c}}{4}\right)\left(p_{b}^{1}-p_{b}^{2}\right)\left(p_{c}^{1}-p_{c}^{2}\right)$ where the indices 1 and 2 refer to the parental populations. Under random crosses, we instructed REALbreeding to generate two descendents by plant (one as male and one as female) and to allow selfing. Under selfing, REALbreeding used the single seed descent process. Thus, the individuals in 
204 generations 0 and the derived inbred lines are non-related and the individuals in generation 10r10s 205 can be related.

206 Based on our input, REALbreeding randomly distributed 10,000 SNPs, 10 QTLs and 90 minor 207 genes (QTLs of lower effect) in 10 chromosomes (1,000 SNPs and 10 genes by chromosome). The 208 average SNP density was $0.1 \mathrm{cM}$. The genes were distributed in the regions covered by the SNPs. 209 Four, three, two, and one QTLs were inserted in chromosomes 1, 5, 9, and 10, respectively. We also 210 specified one SNP within each QTL (with same frequency) and a minimum distance between linked 211 QTLs of $10 \mathrm{cM}$. To allow REALbreeding to compute the phenotypic value for each genotyped 212 individual, we informed the minimum and maximum genotypic values for homozygotes, proportion 213 between the parameter $a$ for a QTL and the parameter $a$ for a minor gene $\left(\mathrm{a}_{\mathrm{QTL}} / \mathrm{a}_{\mathrm{mg}}\right)$, degree of 214 dominance $\left((\mathrm{d} / \mathrm{a})_{\mathrm{i}}, \mathrm{i}=1, \ldots, 100\right)$, direction of dominance, and broad sense heritability. 215 REALbreeding saves two main files, one with the marker genotypes and another with the additive, 216 dominance, and phenotypic values (non-inbred populations) or the genotypic and phenotypic values 217 (inbred populations). The true additive and dominance genetic values or genotypic values are 218 computed from the population gene frequencies (random values), LD values, average effects of 219 gene substitution or $a$ deviations, and dominance deviations. The phenotypic values are computed 220 from the true population mean, additive and dominance values or genotypic values, and from error 221 effects sampled from a normal distribution. The error variance is computed from the broad sense 222 heritability.

223 We simulated three popcorn traits. The minimum and maximum genotypic values of 224 homozygotes for grain yield, expansion volume, and days to maturity were 30 and 180 g per plant, 22515 and $65 \mathrm{~mL} / \mathrm{g}$, and 100 and 170 days, respectively. We defined positive dominance for grain yield $226\left(0<(\mathrm{d} / \mathrm{a})_{\mathrm{i}} \leq 1.2\right)$, bidirectional dominance for expansion volume $\left(-1.2 \leq(\mathrm{d} / \mathrm{a})_{\mathrm{i}} \leq 1.2\right)$, and no 227 dominance for days to maturity $\left((\mathrm{d} / \mathrm{a})_{\mathrm{i}}=0\right)$. The broad sense heritabilities were 40 and $80 \%$. These 228 values can be associated with individual and progeny assessment, respectively. Assuming $\mathrm{a}_{\mathrm{QTL}} / \mathrm{a}_{\mathrm{mg}}$ 
$229=10$, the QTL heritabilities ranged from 3.2 to $11.8 \%$. The GWAS was performed in population 1,

230 generations 0 and 10r10s, and in the inbred lines panel obtained from inbreds of the populations 1

231 through 4, generation 0 (generations 10s). To assess the influence of the sample size on the GWAS

232 efficiency, we considered sample sizes of 400 and 200. Thus, we used 100 or 50 inbreds from

233 populations 1 through 4 to generate the inbred lines panel.

234 Statistical analyses

235 The analysis of the $\mathrm{Q}+\mathrm{K}$ linear mixed model was performed with the software GWASpoly

236 (Rosyara et al. 2016) fitting the additive and additive-dominance models for the open-pollinated

237 population and the additive model for the RILs and inbred lines panel. For the population structure

238 analysis, we used Structure software (Falush et al. 2003) and fitted the admixture model with

239 correlated allelic frequencies and the no admixture model with independent allelic frequencies. The

240 number of SNPs, sample size, burn-in period, and number of MCMC (Markov chain Monte Carlo)

241 replications were 100 (10 random SNPs by chromosome), 400 (simulation 1), 10,000, and 40,000,

242 respectively. The number of populations assumed $(\mathrm{K})$ ranged from 1 to 7 , and the most probable $\mathrm{K}$

243 value was determined based on the inferred plateau method (Viana et al. 2013). The population

244 structure analysis evidenced four subpopulations (data not shown).

245 To classify each significant association as true or false, we used a program developed in

246 REALbasic 2009 by the first author. The classification criterion was based on the difference

247 between the position of the SNP and the position of a true QTL (candidate gene). If the difference

248 was less than or equal to $2.5 \mathrm{cM}$ (Yu et al. 2008), the association was classified as true. The GWAS

249 efficiency was assessed based on the power of QTL detection (probability of rejecting $\mathrm{H}_{0}$ when $\mathrm{H}_{0}$

250 is false; control of the type II error), FDR (control of the type I error), and bias in the estimated

251 QTL position (precision of mapping) (Li et al. 2010). We used Benjamini-Hochberg FDR of 5 and

$2521 \%$ to control the type I error (Benjamini and Hochberg 1995).

\section{Data availability}


REALbreeding is available upon request. The data set is available at 255 https://dx.doi.org/10.6084/m9.figshare.3201838.version3. Supplemental file S1 contains detailed 256 description of all data files (SNP and QTL positions, SNP genotypes, and phenotypic values). Data 257 citation:

258 Viana, José Marcelo Soriano; Mundim, Gabriel Borges; Pereira, Hélcio Duarte; Andrade, Andréa 259 Carla Bastos; Fonseca e Silva, Fabyano (2017): Efficiency of genome-wide association study in 260 random cross populations. figshare. https://dx.doi.org/10.6084/m9.figshare.3201838.version3

\section{RESULTS}

Our first result from the open-pollinated population, assuming sample size of 400 and FDR of $1 \%$, was disappointing since most grain yield QTLs of high heritability showed low power of detection. For example, the power of detection for the QTLs with heritabilities of 8.4, 9.4, and $11.6 \%$ were $4.2,8.3$, and $10.4 \%$, respectively (Figure 1a). We realized that the problem was the high dominance deviation for these QTLs (the greatest values among the 10 QTLs). This explained 267 the relatively low coefficients of determination for the linear regression models relating QTL 268 detection power and heritability, especially with sample size of 200 and FDR of 5\% (45, 55, and 19\%) (Figure1a, c, and Figure 2a). The solution to this problem was to fit the additive-dominance model for grain yield and expansion volume. Regardless of the sample size and FDR, for $76 \%$ of 271 the grain yield and expansion volume QTLs the detection power was increased (Figures 1b, d, and 272 Figure 2b). Previously undetected QTLs showed detection power ranging from 2.3 to 52.3\%. The 273 increase in the detection power for previously detected QTLs ranged from 0.8 to $2,300 \%$ (244.6\% 274 on average), mainly with sample size of 400 and FDR of $1 \%$. A consequence of fitting the additive275 dominance model for grain yield and expansion volume was an increase in the coefficients of 276 determination for the linear regression models relating QTL detection power and heritability, 277 especially with lower sample size ( $\mathrm{R}^{2}$ of 79,81 , and $46 \%$ ) (Figure1b, d, and Figure 2b). Unlike, the 278 additive-dominance model should not be fitted for traits showing no dominance. Fitting the 
279

\section{0}

281

282

283

284

285

286

287

288

289

290

291

292

293

294

295

296

297

298

299

300

301

302

303

additive-dominance model for days to maturity, for $82 \%$ of the QTLs the detection power decreased from 6.6 to $100.0 \%$ (48.4\% on average), regardless of sample size and FDR.

Defining low, intermediate, and high heritability QTLs as those with heritability values less than or equal to $3.5 \%$, between 3.6 and $7.5 \%$, and greater than $7.5 \%$, respectively, the power of detection was maximized increasing the sample size from 200 to 400 and the FDR from $1 \%$ to $5 \%$

(Table 1). It is important to highlight that under sample size of 400 the observed FDR was equal to or lower than the specified level of significance. Assuming sample size of 400 and FDR of 5\%, the average power of detection for the low, intermediate, and high heritability QTLs were 9.7, 32.7, and $87.7 \%$, respectively. The minimum and maximum values were 2 and $29.5 \%, 5.5$ and $94 \%$, and 62.0 and $100.0 \%$. The observed FDR was $3.8 \%$. Decreasing the sample size to 200 decreased the QTL detection power (28, 67 and $62 \%$ for the low, intermediate and high heritability QTLs, respectively) and increased the observed FDR to $9.4 \%$. Concerning the bias in the QTL (candidate gene) position, it should be also highlighted that at least 97\% (assuming sample size of 200 and FDR of 5\%) of the QTLs were declared by the SNP within it, and that the number of significant SNPs within the range of $2.5 \mathrm{cM}$ was very low, regardless of sample size and FDR. The average number of significant SNPs within the range of $2.5 \mathrm{cM}$ varied from 0.1 to 1.0. The average bias in the QTL position from a significant SNP within the range of $2.5 \mathrm{cM}$ varied from approximately 0.2 to $0.4 \mathrm{cM}$. Furthermore, we also observed that the QTL detection power has low correlation with the QTL average effect of substitution (0.1) and QTL dominance deviation (0.2). The correlation between QTL detection power and QTL heritability ranged from 0.68 to 0.90 , proportional to the sample size.

The analysis of the RILs from the open-pollinated population provided essentially the same results (similar magnitude of the statistics) concerning QTL detection power, control of the type I error, and mapping precision (Figure $2 \mathrm{c}, \mathrm{d}$, and Table 1 ). The only significant difference was the absence of low heritability QTLs. We can highlight a slightly better control of the type I error also. 
304 Thus, the QTL detection power was also maximized assuming sample size of 400 and FDR of 5\% 305 and the observed FDR was $1.8 \%$ in this scenario. The decrease in the sample size significantly 306 decreased the power of QTL detection (54 and 65\% for the intermediate and high heritability QTLs, 307 respectively) and increased the FDR (to 7.3\%) too. Regarding of the inbred lines panel, it is 308 impressive to realize that this population provided an improvement in the outstanding results 309 offered by the non-inbred and inbred open-pollinated populations, concerning low and intermediate 310 heritability QTL detection power, control of the type I error, and mapping precision (Figure 3a, b, 311 and Table 1). The increase in the low heritability QTL detection power ranged from 74 to $177 \%$. 312 For the intermediate heritability QTLs the increase ranged from 39 to 64\%. The observed FDR was 313 reduced in up to $72 \%$ and the bias in the QTL position decreased between 80 and $87 \%$. The QTL 314 detection power was also maximized assuming sample size of 400 and FDR of 5\%, combined with 315 an observed FDR of $2.7 \%$. Similarly to non-inbred and inbred open-pollinated populations, the 316 decrease in the sample size significantly decreased the power of QTL detection $(54,61$ and $61 \%$ for 317 the low, intermediate, and high heritability QTLs, respectively) but the FDR was unaffected.

318 Finally, it is important to highlight that the FDR is mainly affected by population structure, 319 compared to relationship information. Assuming non-inbred population, sample size of 400 and 320 FDR of $1 \%$, ignoring the relationship information (by ignoring the polygenic effect), the observed 321 FDR was unaffected (1.1 vs. $1.4 \%$ ) but the number of significant associations outside the $2.5 \mathrm{cM}$ 322 interval was drastically increased (from practically zero to 28; Table 1). As will be discussed 323 further, these are not all false-positive associations but due to LD between the SNPs and one or 324 more QTLs in the chromosome. With RILs, because the level of LD is lower than in the non-inbred 325 population, the observed FDR was $1.2 \%$ but the number of significant associations outside the 2.5 $326 \mathrm{cM}$ interval achieved approximately 7. For inbred lines panel, ignoring only the relationship 327 information the FDR was $0.8 \%$ but ignoring only population structure increased the FDR to $29.5 \%$ 328 Ignoring the relationship information and population structure in the analysis of the inbred lines 
329

panel determined thousands of significant associations in all chromosomes, increasing drastically the FDR (to approximately 60\%; data not shown). In these scenarios it was not possible to detect QTLs because many significant associations were observed along the length of a chromosome or in one or more large chromosome regions, especially for chromosomes 1 (four QTLs) and 5 (three QTLs) (data not shown).

\section{DISCUSSION}

The presented theory proves that a significant association from a GWAS in a non-inbred or inbred random cross population and in an inbred lines panel, correcting for population structure and relatedness, is due to LD between the SNP and one or more linked QTLs. The theory also shows that GWAS provides estimation of the average effect of a SNP substitution (and consequently the estimation of SNP effects). Using SNP effects to measure chromosome and chromosome $\mathrm{x}$ inbred effects, Bernardo and Thompson (2016) showed that GWAS also provide dissection of the germplasm architecture for quantitative traits. Schaefer and Bernardo (2013) estimated SNP effects and identified candidate genes and QTL hot spots (chromosome regions with previously mapped QTLs) for days to flowering, kernel composition, and disease resistance. Based on the theory presented, only if there is a single QTL in LD with a significant SNP, if the SNP is within the QTL, and if QTL and SNP alleles have the same frequency it is adequate to consider the SNP average effect of substitution as the QTL average effect of substitution. We additionally provided the parametric values of SNP effects commonly fitted in the GWAS and genomic selection models, and the genotype and gametic probabilities and the parametric LD values in a completely inbred population and in an inbred lines panel. The LD in a group of RILs are lower than the LD in the non-inbred population and the $\mathrm{LD}$ value in an inbred lines panel tends to be lower than the $\mathrm{LD}$ in each group of inbreds (that are lower than the LD in the base populations) because it is an admixture of positive and negative LD values. 
To our knowledge, this is the first study on GWAS efficiency in open-pollinated population.

354 The results are impressive and show that the identification of candidate genes can be highly

355 efficient, depending on sample size and QTL heritability. LD is for sure another important factor

356 affecting GWAS (Weir 2008). Thus, based on a sample of 400 individuals and defining a level of

357 significance of $5 \%$, the power of detection of low $(\leq 3.5 \%)$, intermediate $(3.6-7.5 \%)$, and high $(\geq$

358 7.6) heritability QTLs can achieve approximately 30, 90, and 100\%, respectively (10, 30, and $90 \%$

359 on average). This result is achieved keeping the FDR bellow 5\% and is associated with a very low

360 number of significant associations close to the QTL (highly precise mapping), besides the

361 significant SNP within the QTL. This means that GWAS efficiency is maximized when there is at

362 least one SNP within each QTL, with the same allelic frequency. This seems very restrictive and,

363 unfortunately, is. To achieve high efficiency when there is not a SNP within each QTL, high LD

364 between a SNP close to the QTL and greater sample size are required, especially for low heritability

365 QTLs. In a random cross population the LD measure depends also on the SNP and QTL allele

366 frequencies. Thus, significant associations involving few SNPs with the same QTL can be observed,

367 including SNPs that are tens of mega base pairs (or centiMorgans) from the QTL. In reality, a

368 closely linked QTL and SNP can have a lower LD value compared to a more distant QTL and SNP

369 pair. In populations with low level of $\mathrm{LD}$, significant associations are expected to occur for only

370 SNPs within the QTL or located very close to the QTL (within a few hundred base pairs), which

371 favors the identification of a candidate gene for the QTL. In this scenario, a QTL would be declared

372 based on one to a small number of significant associations spanning a chromosome region of a few

373 kilo base pairs (not mega base pairs or centiMorgans).

374 Field results have demonstrated that GWAS are best carried out with a large sample size (Yu

375 and Buckler 2006). According to Flint-Garcia et al. (2005), increasing the population size increases

376 the number of individuals with rare alleles, thus improving the power to test the association between

377 these rare alleles and the trait of interest. Yu et al. (2008) showed that the gain in the GWAS 
378 efficiency by increasing sample size was evidenced by increased power of QTL detection and 379 smaller FDR, mainly with heritability of 0.7 in comparison with a heritability of 0.4 . Based on a 380 simulation study, Long and Langley (1999) demonstrated that approximately 500 individuals 381 should be genotyped for 20 SNP loci within the candidate gene region to detect marker-trait 382 associations for QTLs that account for as little as 5\% of the phenotypic variation. They observed 383 that more power was achieved by increasing the population size than by increasing the SNP density 384 within the candidate gene region.

385 Our most significant contribution to the knowledge on GWAS is the empirical proof that the 386 additive-dominance model must be fitted for traits controlled by dominance (uni- or bidirectional), 387 to increase the QTL power of detection. We additionally evidenced that the additive-dominance 388 model must not be fitted for traits determined only by additive gene effects, to avoid a decrease in 389 the QTL detection power. This is probably due to over fitting. Further, we provided results for 390 comparing GWAS in non-inbred and inbred random cross populations, and in an inbred lines panel. 391 The inbreeding did not affect the GWAS efficiency, but RILs, if available, can be interesting to 392 maximize the QTL heritabilities, since they allow standard experimental procedures (local control 393 and replication) and the assessment of SNP x environment interaction. Compared to GWAS in an 394 inbred lines panel, GWAS in random cross population was less efficient, i.e., showed lower power 395 of QTL detection for the low and intermediate heritability QTLs, slightly inferior control of false396 positive associations, and higher bias in QTL position. This is due to higher genetic variability in 397 the inbred lines panel since the average LD in the population 1, generation 0 , is higher than the 398 average $\mathrm{LD}$ in the inbred lines panel (average absolute $\Delta$ equal to 0.0403 and 0.0249 , respectively). 399 The genetic variability in the inbred lines panel is 9 to 13 times greater, depending on the trait (data 400 not shown).

401 According to Flint-Garcia et al. (2005), the inbred lines panel exploits the rapid breakdown of 402 LD in diverse maize lines, enabling very high resolution for QTL mapping. Population structure 
403

404

405

406

407

408

409

410

412

413

414

415

416

417

418

420

421

422

423

424

425

426

427

results from constructing a panel with inbreds from various breeding programs and distinct heterotic groups, which can cause false-positive marker-trait associations if the data is not corrected (Yan et al. 2009). The lowest parametric LD values for the inbred lines panel occurred in published studies (Yan et al. 2009, Remington et al. 2001). Moreover, with the inbred lines panel, generally, only SNP loci within the QTL showed significant association, which is a highlighted result from GWAS that can serve as a basis for a fine mapping strategy for marker-assisted selection and map-based cloning genes (Gupta et al. 2005).

Our results are comparable to previous GWAS with field and simulated data. Concerning the QK model, Bernardo (2013) observed that the power of QTL detection and number of false-positive associations were proportional to the sample size. Assuming FDR of approximately $1 \%$ and an average QTL heritability of approximately 5\%, the power of detection increased from 13 to $45 \%$ when the sample size increased from 384 to 1,536. In the study of Yang et al. (2010) the QTL detection power was relatively low for QTLs with heritability lower than $10 \%$ but increased significantly with the increase in the population size. Assuming sample size of 155 , the power of detection was $16.5,59.2$, and $87.6 \%$ for the low $(1 \%)$, intermediate $(5 \%)$ and high $(\geq 10 \%)$ heritability QTLs, respectively. Yu et al. (2008) investigated the genetic and statistical properties (power of QTL detection and FDR) of the nested association mapping (NAM) design. With 5,000 genotypes, they achieved an average power of QTL detection of $57 \%$ (with a range of 30 to $85 \%$ ) when considering two trait heritabilities (0.4 and 0.7) and two different numbers of QTL controlling the trait (20 and 50). They also observed that a higher heritability always gave higher QTL detection power, particularly for QTL with moderate to small effect. However, the FDR values were high, ranging from 9 to $23 \%$.

Concerning the relevance of relatedness, even if due to identity by state, and population structure correction, our findings agreed with previous knowledge that the best GWAS model must include a polygenic effect - to eliminate significant associations outside of the QTL interval 
428 (including false-positive associations) - and a population structure effect - to control the type I error, 429 as highlighted by Yu et al (2006) and Bernardo (2013), among others. Stich and Melchinger (2009) 430 and Yang et al. (2010) observed best control of spurious associations by the K model. In the study 431 of Flint-Garcia (2005) the population structure effect was significant, explaining 9.3\% of the 432 phenotypic variation, on average.

The GWAS in plant breeding has been effective for identifying candidate genes for 434 quantitative traits such as plant architecture, kernel composition, root development, flowering time, 435 drought tolerance, pathogen resistance, and metabolic processes (Zhu et al. 2008). Our study 436 provided the following additional knowledge: 1) the additive-dominance model must be fitted for 437 traits controlled by dominance effects but must not be fitted for traits controlled only by additive 438 effects, to achieve high power of QTL detection; 2) with sample size of 400 and level of 439 significance of 5\%, the power of detection for the low, intermediate, and high heritability QTLs can 440 achieve approximately 30, 90, and 100\%, respectively; 3) under sample size of 400, the observed 441 FDR was equal to or lower than the specified level of significance; 4) GWAS in random cross 442 populations is highly precise, since at least $97 \%$ of the QTLs were detected by the SNP inside it and 443 the number of significant associations outside of the QTL interval $(2.5 \mathrm{cM})$ is very low; 5) 444 inbreeding does not affect the GWAS efficiency; 6) identity by state is important to control 445 significant associations outside of the QTL interval; and 7) in random cross populations, FDR is 446 mainly affected by population structure, compared to relationship information. Based on our 447 evidence, breeders can employ non-inbred and inbred populations for GWAS while taking into 448 account that the level of LD should be high, the sample size should be higher than that necessary for 449 QTL mapping, and the QTL heritability should be intermediate to high to achieve greater power of 450 QTL detection and precise mapping of candidate genes. 
452 We thank the National Council for Scientific and Technological Development (CNPq), the

453 Brazilian Federal Agency for Support and Evaluation of Graduate Education (Capes), and the

454 Foundation for Research Support of Minas Gerais State (Fapemig) for financial support.

455

456

457

458

459

460

461

462

463

464

465

466

467

468

469

470

471

472

473

474

475

476

\section{LITERATURE CITED}

Azevedo, C. F., M. D. V. Resende, F. F. Silva, J. M. S. Viana, M. S. F. Valente et al., 2015 Ridge, Lasso and Bayesian additive-dominance genomic models. BMC Genet. 16: 105-118.

Barendse, W., A. Reverter, R. J. Bunch, B. E. Harrison, W. Barris et al., 2007 A validated wholegenome association study of efficient food conversion in cattle. Genetics 176: 1893-905.

Benjamini, Y., and Y. Hochberg, 1995 Controlling the false discovery rate: a practical and powerful approach to multiple testing. J. R. Stat. Soc. 57: 289-300.

Bernardo, R., 2013 Genomewide markers for controlling background variation in association mapping. Plant Genome 6.

Bernardo, R., and A. M. Thompson, 2016 Germplasm architecture revealed through chromosomal effects for quantitative traits in maize. Plant Genome 9.

Falush, D., M. Stephens, and J. K. Pritchard, 2003 Inference of population structure using multilocus genotype data: linked loci and correlated allele frequencies. Genetics 164: 1567-1587.

Flint-Garcia, S. A., A. C. Thuillet, J. Yu, G. Pressoir, S. M. Romero et al., 2005 Maize association population: a high-resolution platform for quantitative trait locus dissection. Plant J. 44: 10541064.

Fraszczak, M, and J. Szyda, 2016 Comparison of significant single nucleotide polymorphisms selections in GWAS for complex traits. J. Appl. Genet. 57: 207-213.

Gupta, P. K., S. Rustgi, and P. L. Kulwal, 2005 Linkage disequilibrium and association studies in higher plants: present status and future prospects. Plant Mol. Biol. 57: 461-485.

Hill, W. G., and A. Robertson, 1968 Linkage disequilibrium in finite populations. Theor. Appl. Genet. 38: 226-231. 
Ingvarsson, P. K., and N. R. Street, 2011 Association genetics of complex traits in plants. New Phytol. 189: 909-922.

Kempthorne, O., 1957 An Introduction to Genetic Statistics. John Wiley and Sons Inc., New York.

Li, H., S. Hearne, M. Banziger, Z. Li, and J. Wang, 2010 Statistical properties of QTL linkage mapping in biparental genetic populations. Heredity 105: 257-267.

Long, A. D., and C. H. Langley, 1999 The power of association studies to detect the contribution of candidate genetic loci to variation in complex traits. Genome Res. 9: 720-731.

Pace, J., C. Gardner, C. Romay, B. Ganapathysubramanian, and T. Lübberstedt, 2015 Genome-wide association analysis of seedling root development in maize (Zea mays L.). BMC Genomics 16: $47-58$.

Pearson, T. A., and T. A. Manolio, 2008 How to interpret a genome-wide association study. J. Am. Med. Assoc. 299: 1335-1344.

Rafalski, J. A., 2010 Association genetics in crop improvement. Curr. Opin. Plant Biol. 13: 174180.

Remington, D. L., J. M. Thornsberry, Y. Matsuoka, L. M. Wilson, S. R. Whitt et al., 2001 Structure of linkage disequilibrium and phenotypic associations in the maize genome. PNAS 98: 1147911484.

Rosyara, U. R., W. S. de Jong, D. S. Douches, and J. B. Endelman (2016) Software for genomewide association studies in autopolyploids and its application to potato. Plant Genome 9: doi: 10.3835/plantgenome2015.08.0073.

Schaefer, C. M., and R. Bernardo, 2013 Genome-wide association mapping of flowering time, kernel composition, and disease resistance in historical Minnesota maize inbreds. Crop Sci. 53: 2518-2529.

Stich, B., and A. E. Melchinger, 2009 Comparison of mixed-model approaches for association mapping in rapeseed, potato, sugar beet, maize, and Arabidopsis. BMC Genomics 10: e94. 
502 Thirunavukkarasu, N., F. Hossain, K. Arora, R. Sharma, K. Shiriga et al., 2014 Functional 503 mechanisms of drought tolerance in subtropical maize (Zea mays L.) identified using genome$504 \quad$ wide association mapping. BMC Genomics 15: 1182-1193.

505 Viana, J. M. S., H.-P. Piepho, and F. F. Silva 2016 Quantitative genetics theory for genomic 506 selection and efficiency of breeding value prediction in open-pollinated populations. Sci. Agric. $507 \quad 73: 243-251$.

508 Viana, J. M. S., H.-P. Piepho, and F. F. Silva 2017 Quantitative genetics theory for genomic 509 selection and efficiency of genotypic value prediction in open-pollinated populations. Sci. Agric. $510 \quad 74: 41-50$.

511 Viana, J. M. S., M. S. F. Valente, F. F. Silva, G. B. Mundim, and G. P. Paes, 2013 Efficacy of 512 population structure analysis with breeding populations and inbred lines. Genetica 141: 389-399.

513 Weir, B. S., 2008 Linkage disequilibrium and association mapping. Ann. Rev. Genomics Hum. $514 \quad$ Genet. 9: 129-142.

515 Weir, B., 2010 Statistical genetic issues for genome-wide association studies. Genome 53: 869-875.

516 Yan, J. B., T. Shah, M. Warburton, E. S. Buckler, M. D. McMullen et al., 2009 Genetic 517 characterization of a global maize collection using SNP markers. Plos One 4: e8451.

518 Yang, X., J. Yan, T. Shah, M. L. Warbuton, Q. Li et al., 2010 Genetic analysis and characterization 519 of a new maize association mapping panel for quantitative trait loci dissection. Theor. Appl. $520 \quad$ Genet. 121: 417-431.

521 Yu, J., and E. S. Buckler, 2006 Genetic association mapping and genome organization of maize. 522 Curr. Opin. Biotechnol. 17: 1-6.

523 Yu, J., J. B. Holland, M. D. McMullen, and E. S. Buckler, 2008 Genetic design and statistical 524 power of nested association mapping in maize. Genetics 178: 539-551. 
525 Yu, J., G. Pressoir, W. H. Briggs, I. Vroh Bi, M. Yamasaki et al., 2006 A unified mixed-model

526 method for association mapping that accounts for multiple levels of relatedness. Nat. Genet. 38:

$527 \quad 203-208$.

528 Zhu, C., M. Gore, E. S. Buckler, and J. Yu, 2008 Status and prospects of association mapping in 529 plants. Plant Genome 1: 5-20. 
530 Table 1 Average power of detection $(\%)$ for the low $(\leq 3.5 \%)$, intermediate (3.6-7.5\%), and high $(\geq 7.6 \%)$ heritability QTLs, false discovery rate $(\%)$, 531 correlations between power and QTL effect of substitution, dominance deviation, and heritability, bias in the QTL position for significant associations 532 outside the QTL (cM), and number of significant SNPs outside the $2.5 \mathrm{cM}$ interval, regarding population 1, generations 0 (open-pollinated) and 10r10s 533 (RILs), and an inbred lines panel, five models, two sample sizes, and two levels of significance

\begin{tabular}{|c|c|c|c|c|c|c|c|c|c|c|c|c|}
\hline \multirow[t]{2}{*}{ Population } & \multirow[t]{2}{*}{ Model } & \multirow{2}{*}{$\begin{array}{c}\text { Sample } \\
\text { size }\end{array}$} & \multirow{2}{*}{$\begin{array}{l}\text { Sig. } \\
\text { level }\end{array}$} & \multicolumn{3}{|c|}{ Power of detection } & \multirow[t]{2}{*}{ FDR } & \multicolumn{3}{|c|}{ Correlation with power } & \multirow[t]{2}{*}{ Bias } & \multirow{2}{*}{$\begin{array}{l}\text { Sig. } \\
\text { assoc. }\end{array}$} \\
\hline & & & & Low & Int. & High & & Eff. sub. & Dom. dev. & Herit. & & \\
\hline \multirow[t]{7}{*}{ Open-pollinated } & Add.-Dom. ${ }^{\mathrm{a}}$ (K model) & 400 & $1 \%$ & 4.4 & 23.6 & 79.0 & 1.13 & 0.093 & 0.213 & 0.886 & 0.16 & 0.1 \\
\hline & & & $5 \%$ & 9.7 & 32.7 & 87.7 & 3.76 & 0.140 & 0.234 & 0.901 & 0.37 & 1.0 \\
\hline & & 200 & $5 \%$ & 7.0 & 10.8 & 33.2 & 9.44 & 0.097 & 0.227 & 0.680 & 0.20 & 0.6 \\
\hline & Add. (K model) & 400 & $1 \%$ & 9.3 & 18.8 & 51.9 & 0.25 & -0.106 & -0.285 & 0.672 & 0.07 & 0.1 \\
\hline & & & $5 \%$ & 8.2 & 24.2 & 62.7 & 1.57 & -0.079 & -0.245 & 0.740 & 0.13 & 0.2 \\
\hline & & 200 & $5 \%$ & 10.7 & 7.8 & 19.8 & 8.64 & -0.114 & -0.252 & 0.438 & 0.06 & 0.2 \\
\hline & Add. -Dom. ${ }^{\mathrm{a}}$ (K = I model $)$ & 400 & $1 \%$ & 7.1 & 36.9 & 81.0 & 1.42 & 0.074 & 0.164 & 0.686 & 0.91 & 28.3 \\
\hline \multirow[t]{4}{*}{ RILs } & Add. (K model) & 400 & $1 \%$ & - & 22.4 & 80.9 & 0.12 & 0.008 & - & 0.940 & 0.17 & 0.0 \\
\hline & & & $5 \%$ & - & 30.3 & 89.3 & 1.83 & -0.022 & - & 0.954 & 0.33 & 0.2 \\
\hline & & 200 & $5 \%$ & - & 13.9 & 31.3 & 7.30 & -0.007 & - & 0.697 & 0.24 & 0.3 \\
\hline & Add. (K = I model) & 400 & $1 \%$ & - & 33.1 & 82.4 & 1.16 & 0.022 & - & 0.871 & 0.82 & 6.7 \\
\hline \multirow[t]{5}{*}{ Inbred lines panel } & Add. $(\mathrm{Q}+\mathrm{K}$ model $)$ & 400 & $1 \%$ & 12.2 & 35.4 & 85.2 & 0.36 & 0.030 & - & 0.977 & 0.02 & 0.1 \\
\hline & & & $5 \%$ & 26.5 & 45.6 & 93.6 & 2.73 & 0.021 & - & 0.985 & 0.05 & 0.2 \\
\hline & & 200 & $5 \%$ & 12.2 & 17.7 & 36.4 & 2.61 & -0.029 & - & 0.793 & 0.04 & 0.2 \\
\hline & Add. $(\mathrm{Q}+\mathrm{K}=\mathrm{I}$ model $)$ & 400 & $1 \%$ & 15.3 & 37.4 & 87.9 & 0.80 & 0.029 & - & 0.974 & 0.18 & 0.4 \\
\hline & Add. (K model) & 400 & $1 \%$ & 24.7 & 41.4 & 91.0 & 29.5 & 0.002 & - & 0.979 & 0.01 & 0.1 \\
\hline
\end{tabular}

${ }^{\mathrm{a}}$ For grain yield and expansion volume. 


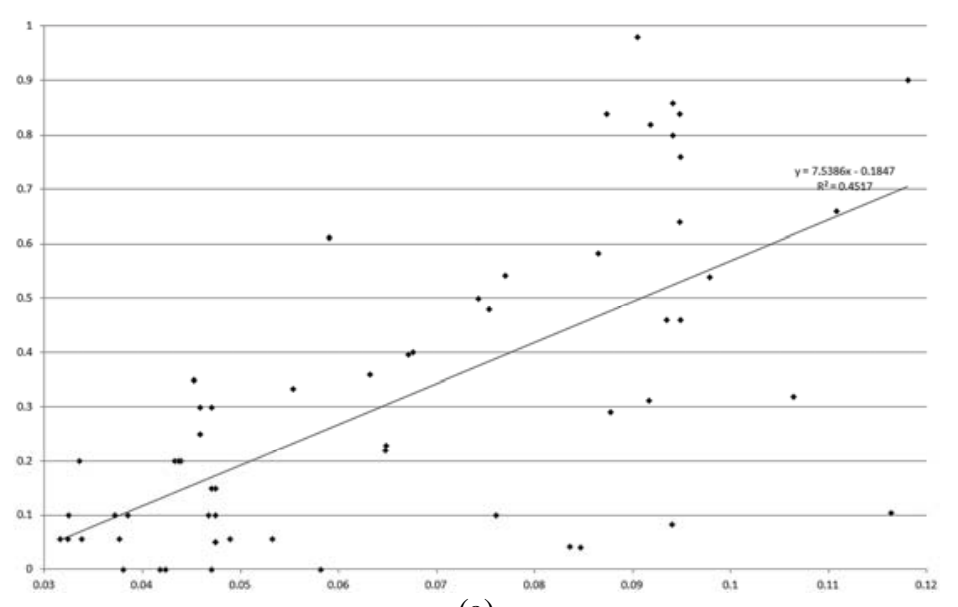

(a)

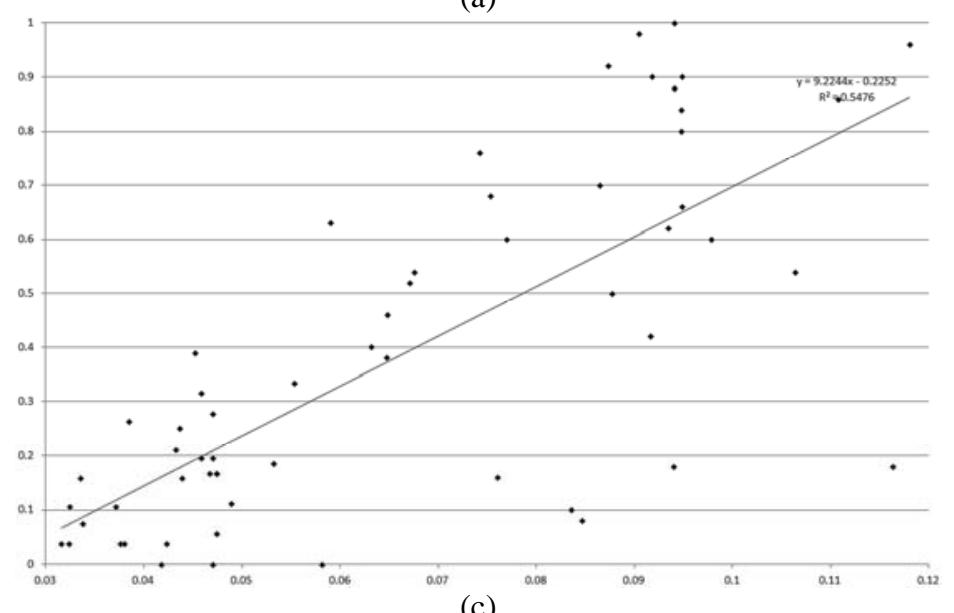

(c)

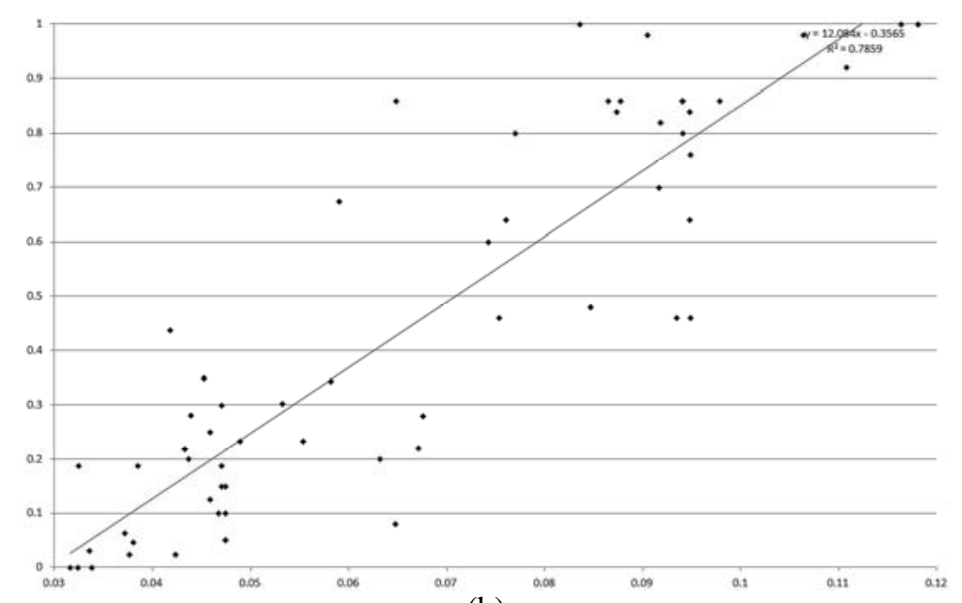

(b)

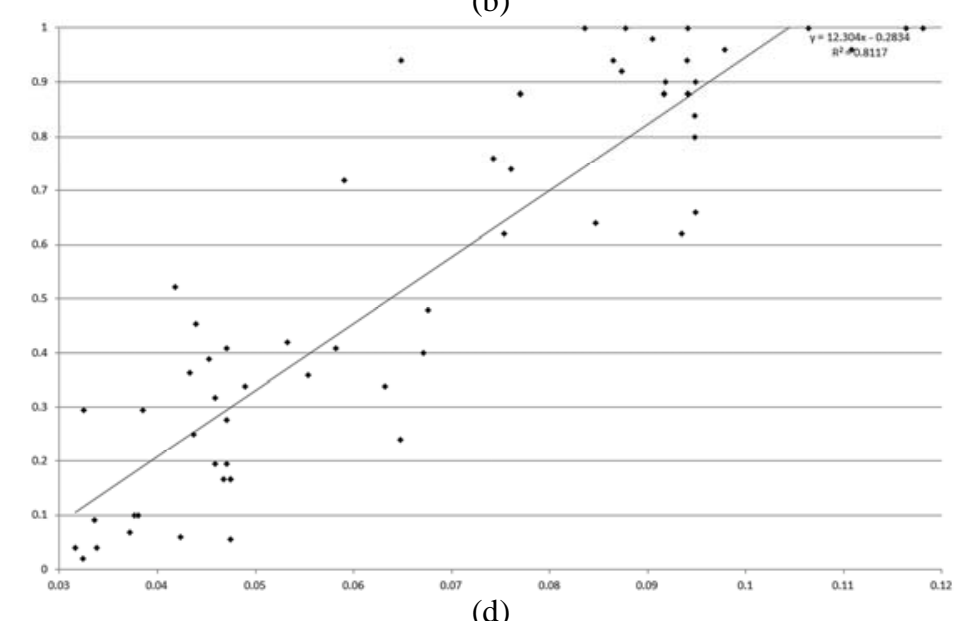

(d)

534 Figure 1 Relationship between QTL heritability (X axe) and power of detection (Y axe) concerning population 1, generation 0, and QTLs determining 535 grain yield, expansion volume, and days to maturity, assuming additive (a, c) and additive-dominance models (b, d), sample size of 400, and levels of 536 significance of $1(\mathrm{a}, \mathrm{b})$ and $5 \%(\mathrm{c}, \mathrm{d})$. 


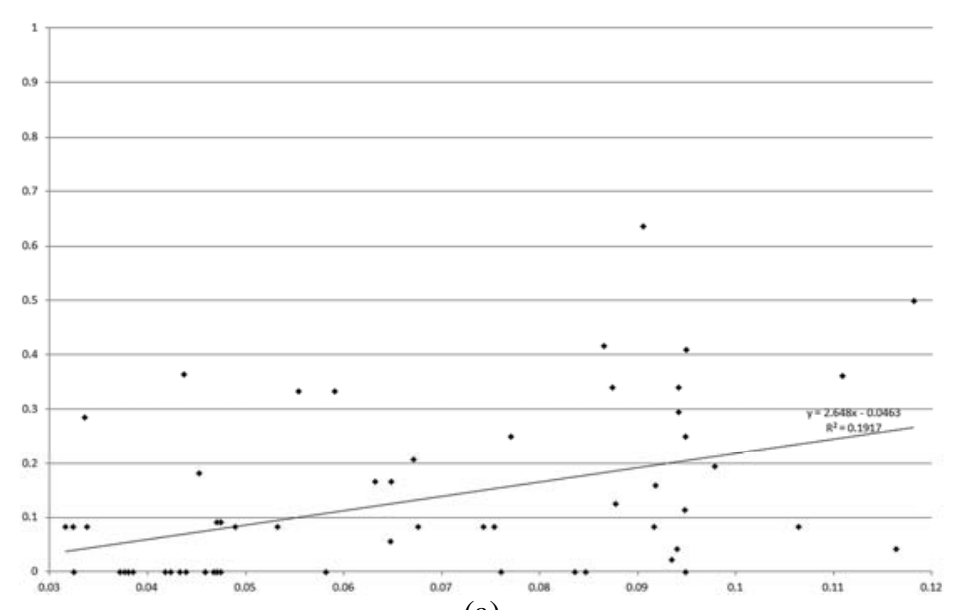

(a)

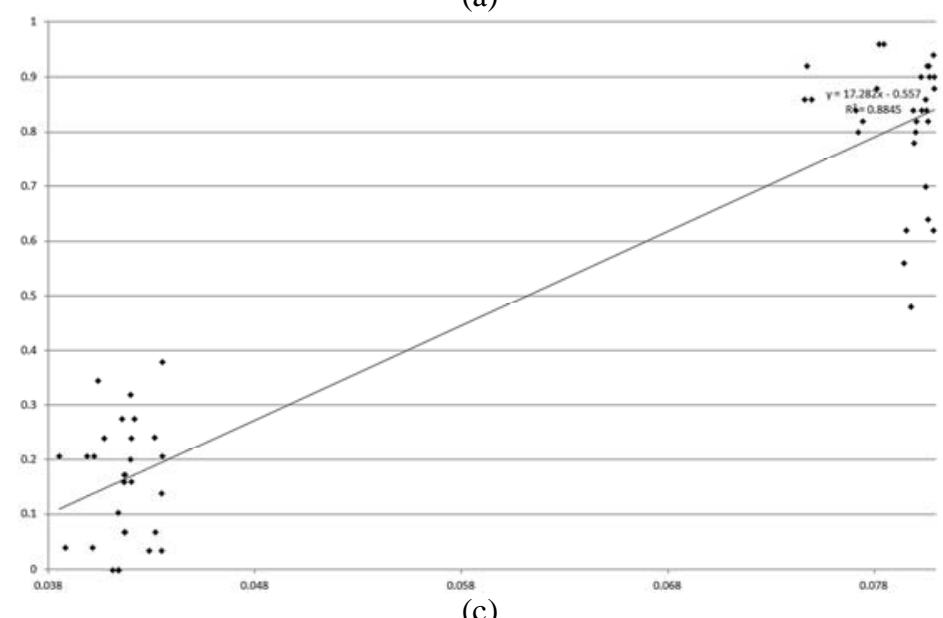

(c)

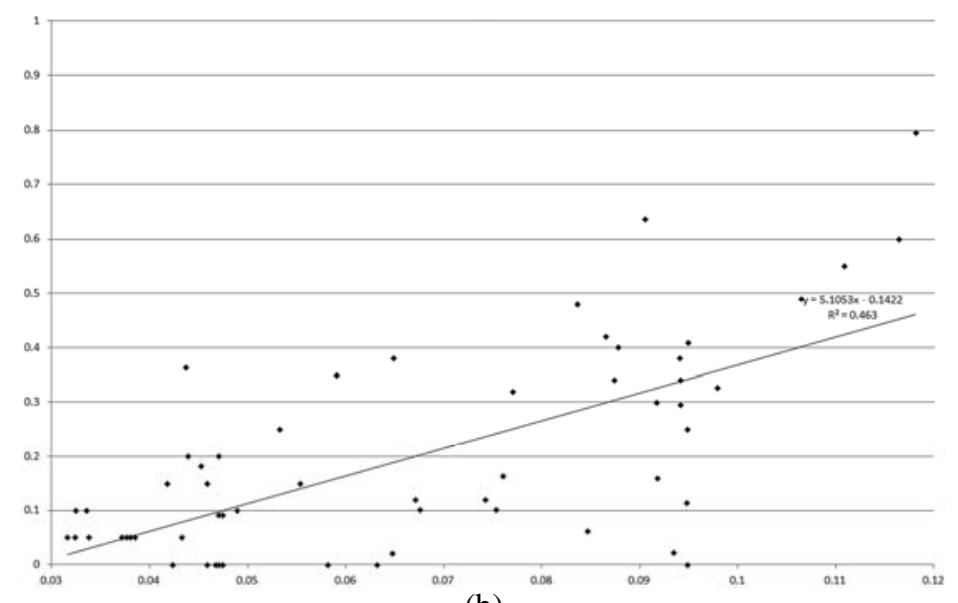

(b)

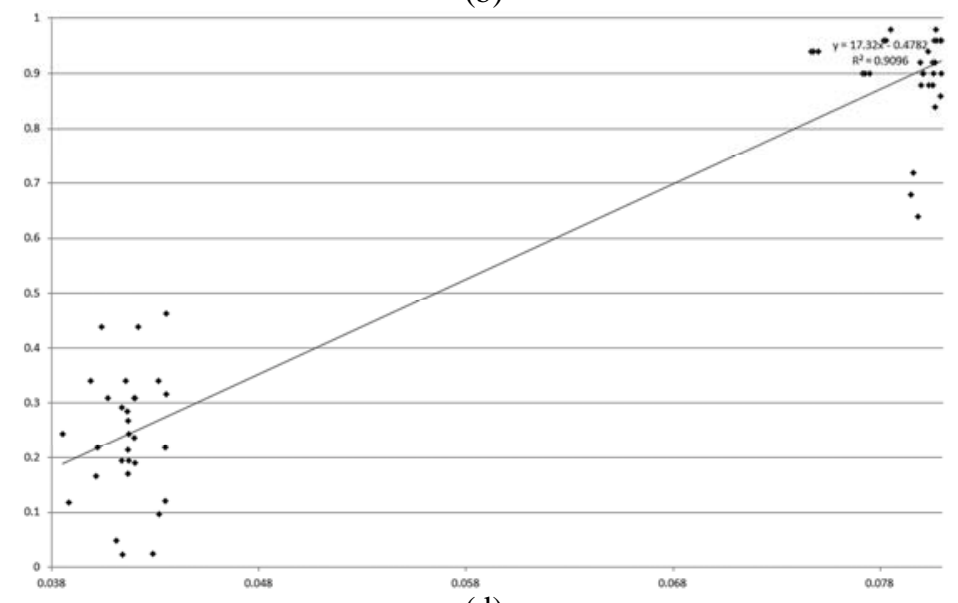

(d)

537 Figure 2 Relationship between QTL heritability (X axe) and power of detection (Y axe) concerning population 1, generations 0 (a, b) and 10r10s (c,

$538 \mathrm{~d}$ ), and QTLs determining grain yield, expansion volume, and days to maturity, assuming additive (a, c, d) and additive-dominance models (b), sample sizes of 400 (c, d) and 200 (a, b), and levels of significance of 1 (c) and 5\% (a, b, d). 


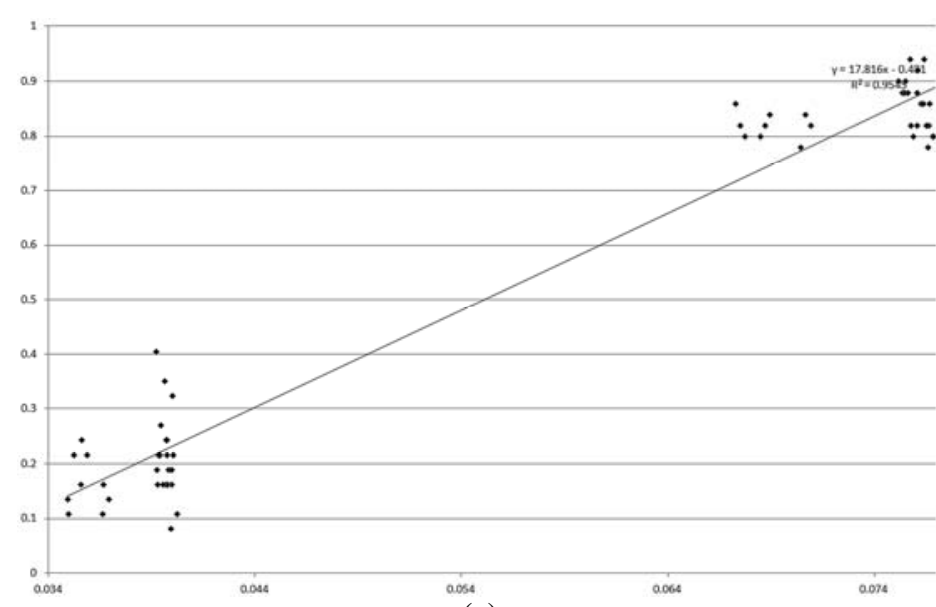

(a)

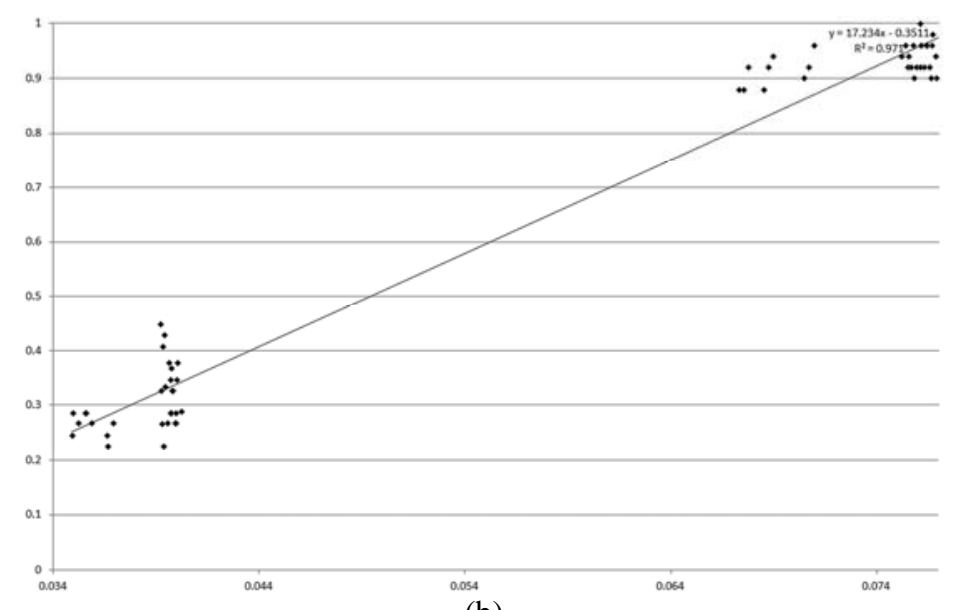

(b)

540 Figure 3 Relationship between QTL heritability (X axe) and power of detection (Y axe) concerning the inbred lines panel and QTLs determining grain 541 yield, expansion volume, and days to maturity, assuming sample size of 400 and levels of significance of 1 (a) and 5\% (b). 